\title{
Gli elaboratori elettronici nel controllo dei voli spaziali $(*)$
}

\author{
R. Pennacchi
}

Ricevuto il 19 Febbraio 1963

Riassunto. - Viene esposta l'organizzazione dei centri di calcolo digitale utilizzati per il controllo dei voli orbitali, in particolare il Goddard Space Center ed il Bermuda Center.

È anche indicato lo schema di calcolo per la determinazione del piano di volo e dei parametri orbitali.

Summary. - The Author exposes the organization of the digital calculation centres utilized for the control of orbital flights, with special regard to the Goddard Space Center and the Bermuda Center.

The calculation scheme, for the determination of the flight plan and of the orbital parameters, is also shown.

\section{Premessa.}

Il problema della realizzazione dei voli spaziali ̀̀, forse, la battaglia più estesa nella quale la scienza si sia mai impegnata, nella sua continua sfida all'univer'so.

Si può dire che tutti i campi della conoscenza vi partecipino con pari importanza: dalla fisica all'ingegneria, dalla biologia all'astronomia, dalla logica alle scienze di organizzazione industriale, alla cibernetica, alle matematiche più elevate.

Ed al centro di questo immenso panorama sta l'uomo, con la sua fragilità fisica, ma sorretto dalle forze formidabili del suo ingegno e della sua volontà.

Ma proprio per la vastitì dei problemi che intervengono nella realizzazione dei voli spaziali, mai si sarebbe potuti passare dalla fase degli

$\left.{ }^{*}\right)$ Nota presentata al $2^{\circ}$ Congresso Internazionale Tecnico Scientifico dello Spazio. Roma, 19-23 Giugno 1962. 
studi teconici ad una effettiva attuazione se l'uomo non aresse avuto al suo servizio non soltanto i mezzi più progrediti per l'esecuzione della parte più materiale dei lavori occolrenti, ma anche quegli esecutori veloci ed infallibili dei lavori più concettuali, che sono i sistemi elettronici per la claborazione e lo snistamento dei dati.

Son i nostra intenzione trattare qui, in forma teorica, quali siano le teconiche impiegate ed i punti nei quali interviene l'elaboratore elettronireo in una organizzazione per lo studio, la progettazione ed il lancio di un veicolo spaziale; vorremmo invece descrivere alcuni esempi di appliazioni concrete che, in questo campo, sono state determinanti nella realizzazione di imprese che hamno stupito il mondo, quali il progetto Mereury il progetto F(ho, il progetto Titan, etc.

Il concetto generale di elaboratore elettronico è ormai troppo familiare perché sia necessario ricordare qui, quali siano i suoi fondamenti logici, le sue unità componenti, il suo morlo di operare.

Ma per questa specifica applicazione, i mezzi che vengono richiesti alla tecmologia sono di natura affatto particolare: in questo campo quelle soluzioni che, nei sistemi elettronici destinati alle normali applicazioni commerciali o scientifiche, possono apparie come troppo complesse o addirittura arveniristiche, risultano attuali ed indispensabili.

In questo campo, si richiedono sistemi (apaci di stolgere le loro funzioni non più limitando le loro elaborazioni a dati provenienti da rilevazioni ed esperienze passate, ma devono essere in grado di operare su un fenomeno fisico che à in atto, e di cui il sistema deve eflettuare un costante controllo, anticipando a preverlendone gili sviluppi futuri ed offiendo la possibilità di intervenire nel fenomeno stesso prima ancora che esso sia giunto alla sua ronclusione.

È questa la situazione tipica che si presenta nel controllo di un veicolo orbitante intorno alla term: compito questo tanto più diammatico quando il veicolo trasporti esseri umani.

\section{LE FASI DI WXA HISSIONE SPIZIALE.}

Per meglio chiarire quali siano i compiti svolti da un sistema elettronico per l'elaborazione dei dati, nel controllo di un volo spaziale, riepiloghiamo qui brevemente le diverse fasi, nel tempo, di un tal genere di missione, prendendo a modello quanto è stato realizzato negli Stati Úniti per il progetto Mereurr: 
a) Pre-lancio. - Comprende un periodo di circa 4 settimane precedenti la partenza del veicolo, ed è dedicato a tutte le operazioni di controllo. Durante questa fase, vengono eseguite tutte le prove necessarie ad ottenere la certezza intorno al perfetto funzionamento di tutti gli elementi e di tutti i componenti della base di lancio, del veicolo e, principalmente, delle apparechiature di comunicazione e di calcolo che costituiscono il sistema nervoso della intera impresa.

b) Lancio. - Va dall'istante di accensione dei reattori fino al momento in cui si può stabilire se il veicolo si sia inserito o no nella orbita prefissatal.

Nel caso del progetto Mercury, dopo il periodo di getto attivo, un sistema elettronico IBM tipo 7090 era in grado di fornire al centro di controllo Nercury di Cape Canaveral una risposta circa la alternativa go - no go.

Isa decisione di Cape Canaveral di rontinuare o interrompere la missione doveva segnare la fine della fase di lancio.

c) Intermzione. - In termini di possibilità, una interruzione della fase di lancio inizia con la segnalazione da parte del sistema elaborante, di una qualche anomalia di carattere cinematico o dinamico e si conclude con un tempestivo azionamento dei retrorazzi.

Naturalmente, possono essere prese decisioni di interruzione a vari livelli: durante l'inizio o alla fine del lancio, secondo il funzionamento dei vari stadi.

In questa fase, il compito principale del calcolatore elettronico è quello di prevedere il tempo ed il punto di impatto in base alle nuove condizioni che si sono verificate.

d) Orbita. - Inizia dall'accertamento che il veicolo è entrato in un'orbita accettabile e dura finché non vengono messi in azione i retrorazzi. In questa fase il calcolatore determina le variabili cinematiche del veicolo, le confronta con le analoghe variabili pre-calcolate, determina il probabile punto e tempo di impatto.

e) Rientro. - Comprende il tempo che va dall'istante di attivazione dei retrorazzi di frenata fino all'atterraggio (o ammaraggio) della cabina spaziale. Durante tutta la fase il calcolatore definisce la posizione attuale del veicolo e calcola, approssimandolo continuamente, il punto di impatto.

f) Recupero. - Questa fase si estende dallatterraggio del veicolo fino a che l'astronauta (o, in generale, il carico trasportato) non sono stati recuperati. 
3. FI'HLABORAZIONE DEI D.ITT XEL PROGETTO MERCLRY.

Sel quarlo generale dell'organizzazione del progetto Mereury, culminato, com'è noto, con il volo orbitale del Maggiore Glenn, furono affidati alla IBM i compiti inerenti l'elabolazione dei dati, compiti che si precisavano nei punti seguenti:

- sviluppo di un metodo di simulazione per le prove dellintero sistema e per l'addestramento del personale;

- sviluppo di un sistema antomatico per il controllo di efficienza durante il "count down " nella fase di pre-lancio;

- sviluppo e costruzione di speciali apparecehiature da calcolo per la raccolta, l'organizzazicne, la trasmissione e l'esposizione in tempo reale dei dati di posizione rilevati dagli apparati sensibili (radars, teodoliti) costituenti la cintur'a di avvistamento;

- impiego di un sistema integrato per la elabolazione in tempo reale dei dati di rilevamento del satellite durante le fasi di orbita e di rientro;

- trasmissione dei dati ai eentri di raccolta per il calcolo delle orbite intorno alla terra, per il loro aggiomamento istantaneo e per la rappresentazione in tempo reale.

I'intero sistema era costituito da una cintura di avistamento comprendente radars a teodoliti installati in varie basi distribuite intorno alla terra, da un sistema di trasmissione digitale ad alta velocità per convogliare i dati rilevati ai centri di elaborazione, da un sistema di elabolazione costituito da 7090 IBM e 709 IBMI installati presso tre centri:

- Mereury Control Center in Cape Canaveral

- Godlard Center in Beltsville

- Bermude Center

Completava il sistema un insieme di apparecchiature per la lappresentazione in tempo reale, destinato a esporre con continuità, in forma urafica e numerica, le risultanze dell'intera missione agli ufficiali della NASA, agli scienziati ed ai tecnici.

Durante tutta la durata della missione, i centri di rilevazione, i centri di controllo ed i centri di elaborazione a calcolo, scambiavano fra loro informazioni, dati e risultati di elaborazioni attraverso quattro linee di trasmissione ad alta velocità (1000 bits al secondo) con messaggi staindard di 408 bits, alla cadenza di ogni mezzo secondo durante il lancio, di 
ogni 6 secondi durante la fase orbitale e di ogni 3 secondi durante il rientro.

Due sistemi tipo 7090 IBM, installati presso il Goddard Center, costituivano il rentro principale di calcolo e di smistamento dei dati.

Questa enorme massa di informazioni poteva essere elaborata in tempo reale dai due sistemi, grazie alla loro elevatissima velocita operativa, di cui puo darsi un'ordine di grandezza considerando che il tempo impiegato per effettuare una somma (con addendi di 10 cifre) è di 218 rentesimi di milionesimo di secondo.

\section{t. RHLEVAZIONE DEL SATEILITE E JELLA SUA ORBI'L.}

a) Impostazione generale del calcolo.

Si suppone esistente un sistema di stazioni di rilevazione. Di queste, però, si considereranno solo quella che si trova alla minima distanza dal punto $J_{1}$ rilevato inizialmente.

Il aalcolo completo verrà impostato attraverso fasi successive, e (ioc:

1. Determinazione del piano dell'orbita;

․ Determinazione dei parametri orbitali.

In questo calcolo proliminare si suppora che il punto $1^{\circ}$ sia molto prossimo al piano $\tau_{1 /}$ contenente l'orbita del satellite.

b) Determinazione del piano dellorbita.

Riferiremo tutti i punti considerati ad un sistema di coordinate artesiane ortogonali aventi l'origine nel centro della terra (che si indicherà con $M$ ) e l'asse $Z$ passante per la stazione di rilevazione $S$ consideratu. Siano $(0,0, \zeta)$ le coordinate di $S$ rispetto al sistema di riferimento prescolito.

Per ciascono dei punti $P_{t}$ rilevati, la stazione $S$ misurerà:

$=$ la distanzal $S P_{i}=d_{i}$

$=$ l'angolo $\psi_{i}$ formato fra la verticale in $S$ e la retta $S P i$

$=$ l'angolo $q_{i}$ (he il piano $S M P_{i}$ forma col piano $x=0$.

Indicate con $\left(x_{i}, y_{i}, z_{i}\right)$ le coordinate - per ora incognite - del punto $P_{i}$ rispetto al riferimento fissato, si avrà:

$$
d_{i}=\sqrt{x_{i}^{2}+y_{i}^{2}+\left(z_{i}-\zeta\right)^{2}}
$$


Inoltre, poiché la verticale in $s$ ha l'equazione

$$
\left\{\begin{array}{l}
x=0 \\
y=0
\end{array}\right.
$$

e la retta $S P_{i}$ ha l'equazione

$$
\left\{\begin{array}{l}
x_{i}=\frac{x_{i}}{z_{i}-\zeta}\left(z-z_{i}\right)+x_{i} \\
y=\frac{y_{i}}{z_{i}-\zeta}\left(z-z_{i}\right)+y_{i}
\end{array}\right.
$$

si ricava, da una nota formula di geometria analitica,

$$
\cos \psi_{i}=\frac{1}{\sqrt{\lambda_{i}^{2}+\mu_{i}^{2}+1}}
$$

ove ì

$$
\begin{aligned}
& \lambda_{i}=\frac{x_{i}}{z_{i}-\zeta} \\
& \mu_{i}=\frac{y_{i}}{z_{i}-\zeta} .
\end{aligned}
$$

Ricordando la [1], la [4] si scrive

$$
\cos \psi_{i}=\frac{z_{i}-\zeta}{d_{i}}
$$

da cui si ricava subito

$$
z_{i}=\zeta+i_{i} \cos \psi_{i}
$$

Il piano $S M P_{i}$ ha l'equazione

ossia

$$
\left|\begin{array}{ccc}
x & y & z \\
0 & 0 & \zeta \\
x_{i} & y_{i} & z_{i}
\end{array}\right|=0
$$

$$
x y_{i}-y x_{i}==0
$$

e quindi l'angolo fra esso ed il piano $x=0$ è dato da

da cui

$$
\cos \varphi_{i}=\frac{y_{i}}{\sqrt{y_{i}^{2}+x_{i}^{2}}}
$$

$$
\cos ^{2} \varphi_{i}\left(y_{i}^{2}+x_{i}\right)=y_{i}^{2}
$$

ossia

$$
\cos ^{2} \varphi_{i}\left[d_{i}^{2}-\left(z_{i}-\zeta\right)^{2}\right]=y_{i}^{2} .
$$


Si laanno rosi le tre equazioni

$$
\begin{aligned}
& x_{i}^{2}+y_{i}^{2}+\left(z_{i}-i_{i}^{2}=d_{i}^{2}\right. \\
& z_{i}=\zeta+d_{i} \cos \psi_{i} \\
& y^{2}+\left(z_{i}-\zeta\right)^{2} \cos ^{2} \varphi_{i}=d_{i}^{2} \cos ^{2} \varphi_{i}
\end{aligned}
$$

da cui si ricava

$$
\begin{aligned}
& z_{i}=\zeta+d_{i} \cos \psi_{i} \\
& y_{i}=d_{i} \cos \varphi_{i} \operatorname{sen} \psi_{i} \\
& x_{i}=d_{i} \operatorname{sen} \varphi_{i} \operatorname{sen} \psi_{i}
\end{aligned}
$$

Rilevando successivamente due punti $P_{1}$ e $P_{2}$, è allora determinabile il piano $\pi_{0}$ passante per $P_{1}, P_{2}$, ed $M$.

Tale piano, che contiene l'orbita ellittica del missile, avrà l'ecquazione

$$
\left|\begin{array}{ccc}
x & y & z \\
x_{1} & y_{1} & z_{1} \\
x_{2} & y_{2} & z_{2}
\end{array}\right|=0
$$

con $x_{1}, y_{1}, z_{1}$ e $x_{2}, y_{2}, z_{2}$ forniti dalle $[15]$ per $i=1,2$, ossia

$$
\alpha x+\beta y+\gamma z=0
$$

(o)

$$
\begin{aligned}
& \alpha=y_{1} z_{2}-y_{2} z_{1} \\
& \beta=x_{2} y_{1}-x_{1} y_{2} \\
& \gamma=x_{1} y_{2}-x_{2} y_{1} .
\end{aligned}
$$

\section{c) Jeterminazione dell'orbita del satellite.}

Individuato il piano $\pi_{0}$ contenente l'orbita del satellite, è possibile, rile vando un terzo punto $P_{3}$, determinare l'orbita stessa.

Consideriamo, infatti, un secondo sistema di riferimento $M\left(x^{\prime}\right.$, $\left.y^{\prime}, z^{\prime}\right)$ avente come piano

$$
z^{\prime}=0
$$

il piano $\pi_{0}$, come asse $y^{\prime}\left(x^{\prime}=z^{\prime}=0\right)$ la retta passante per il centro della terral $M$ e per il punto $I$ di intercettazione (che si suppone contenuto in $\pi_{0}$ ed avente le coordinate $x_{I}, y_{I}, z_{I}$ e come asse $x^{\prime}\left(y^{\prime}-z^{\prime}-0\right)$ la normale in $M$ alla retta $M I$ (e contenuta in $\pi_{0}$ ). 
Se le tre rette $x^{\prime}, y^{\prime}, z^{\prime}$ hanno in $M(x, y, z)$ le equazioni

$$
\begin{array}{ll}
\frac{x}{l_{1}}=\frac{y}{m_{1}}=\frac{z}{n_{1}} & \text { per l'asse } x^{\prime} \\
\frac{x}{l_{2}}=\frac{!}{m_{0}}=\frac{z}{m_{0}} & \text { jer l'asse } !^{\prime} \\
\frac{x}{l_{1}}=\frac{!}{m_{n}}=\frac{z}{m_{2}} & \text { jer l'asse } z^{\prime}
\end{array}
$$

dalle rondizioni soplä indirate si ricava:

1) per l'asse ?'

$$
\begin{aligned}
& l_{0}=l_{2} x_{I}=\cos \hat{y^{\prime} x} \\
& m_{2}=l_{2} y_{l}=\cos \hat{y^{\prime} !} \\
& h_{2}=l_{2} z_{I}=\cos \hat{y^{\prime} z}
\end{aligned}
$$

(he impongono il passaggrio della [21] per $I$;

2) per l'asse $z^{\prime}$

$$
\begin{aligned}
& l_{3}=l_{i_{3}} \alpha=\cos z^{\prime} x \\
& m_{3}=l_{3} \beta=\cos z^{\prime} y \\
& m_{3}=l_{3} \gamma^{\prime}=\cos z^{\prime} z
\end{aligned}
$$

(he impongono la normalità al piano $\pi_{t}$;

3) per' l'asse $x^{\prime}$

$$
\begin{aligned}
& l_{1} l_{2}+m_{1} m_{2}+n_{1} n_{2}=0 \\
& l_{1} l_{3}+m_{1} m_{3}+n_{1} n_{3}=0
\end{aligned}
$$

(che impongono la normalità alle due rette $y^{\prime}$ a $z^{\prime}$.

Dalle [25] si ricava

$$
\begin{aligned}
& l_{1}-l_{1}\left(m_{2} n_{3}-m_{3} n_{2}\right)=\cos \widehat{x^{\prime} x} \\
& m_{1}=l_{1}\left(l_{3} n_{2}-l_{2} n_{3}\right)=\cos x^{\prime} y \\
& n_{1}=l_{1}\left(m_{3} l_{2}-m_{2} l_{3}\right)=\cos x^{\prime} z .
\end{aligned}
$$

Ciò posto ì facile determinare le formule di trasformazione whe permettono il passagerio dal sistema $M(x, y, z)$ al sistema $M\left(x^{\prime}, y^{\prime}, z^{\prime}\right)$. Infatti i

$$
\begin{aligned}
& x=x^{\prime} l_{1}+y^{\prime} l_{2}+z^{\prime} l_{3} \\
& y=x^{\prime} m_{1}+y^{\prime} m_{2}+z^{\prime} m_{3} \\
& z=x^{\prime} n_{1}+y^{\prime} n_{2}+z^{\prime} n_{3}
\end{aligned}
$$


e, inver'samente

$$
\begin{aligned}
& x^{\prime}=l_{2} x+m_{1} y+n_{1} z \\
& y^{\prime}=l_{2} x+m_{0} n+n_{2} z \\
& z=l_{3} x+m_{3} y+n_{3} z
\end{aligned}
$$

E ovio, come si ricuva dalle [24], che se un punto considerato giace sul piano $\pi_{0}$, per esso sarà $z^{\prime}=0$.

Consideriamo ora, nel piano $\pi_{0}$, il sistema di jiferimento $M\left(x^{\prime}, y^{\prime}\right)$.

In esso i punti $P_{1}$ e $P_{2}$ avranno le coordinate $\left(x_{1}, y_{1}^{\prime}\right)$ e $\left(x_{2}^{\prime}, y_{2}^{\prime}\right)$ date dalle [28]. Consideriamo anche un terzo punto rilevato, avente coordinate $\left(x_{3}, y_{3}, z_{3}\right)$ lispet to al sistema $M(x, y, z)$ a $M\left(x_{3}^{\prime}, y_{3}^{\prime}, 0\right)$ rispet to al sistema $M\left(x^{\prime}, y^{\prime}, z^{\prime}\right)$.

I tre punti $P_{1}, P_{2}, P_{3}$, dorendo appartenere ad una ellisse con fuoco in $M$, determinano l'ellisse stessa che avrà, rispetto al riferimento $M$ ( $x^{\prime}$, $\left.y^{\prime}, z^{\prime}\right)$ le equazioni

$$
\left\{\begin{array}{l}
\sqrt{x^{\prime 2}+y^{2}}=4 x^{\prime}+B y^{\prime}+C \\
z^{\prime}=0
\end{array}\right.
$$

I coefficienti $A, B$, ('possono ricararsi imponendo le tre condizioni di passaggrio per $P_{1}, P_{2}, P_{3}$.

Allora la quota di passaggio del missile sulla verticale di $I$ sarà data dall'intersezione della ellisse [297 con l'asse $y^{\prime}$, ossia da

$$
\begin{aligned}
& \pm y^{\prime}=B y^{\prime}+c \\
& z^{\prime}=0 \\
& x^{\prime}=0
\end{aligned}
$$

ossia dai due punti

$$
\left\{\begin{array} { l } 
{ y ^ { \prime } = \frac { C } { 1 - B } } \\
{ x ^ { \prime } = z ^ { \prime } = 0 }
\end{array} \quad \left\{\begin{array}{l}
y^{\prime}=-\frac{C}{1+B} \\
x^{\prime}=z^{\prime}=0 .
\end{array}\right.\right.
$$

Scartando il secondo ad indiando con $R$ il raggrio dellat terria (o meglio la distanzal $I M)$, la quota cercata salrà

$$
z_{j}=\frac{C}{1-B}-R
$$




\section{I'GLABORMZIONF DEI DATI IN TEMPO REALE.}

In tutte le fasi della missione Mercury, era di vitale importanza che i differenti e numerosi tipi di calcoli richiesti fossero eseguiti con precisione assoluta e resi disponibili istantanenmente, e questa richiesta si imponeva come necessità fondamentale per la sicurezza dell'astronauta ed il successo dell'intero progetto.

Per dare un'idea del volume di calcoli da sviluppare, si pensi, ad esempio, che nei primissimi istanti dell'inserimento del veicolo in orbita era necessario calcolare i parametri dell'intera orbita, allo scopo di decidere se la missione poteva proseguire oppure era necessario interromperla.

Considerando che la rotazione di 180 gradi del veicolo, necessaria per l'azionamento del retrorazzi di freno, richiedeva un certo tempo e che il punto di impatto d'emergenza doveva essere sul mare, si vede facilmente quanto breve era il tempo a disposizione per il calcolo dei parametri orbitali.

Perciò fin dalla fase di lancio, i sistemi 7090 IBM del Goddard Center effettuir'ono i calcoli necessari a decidere, in ogni istante, l'alternativa go - no go, mediante la determinazione dei parametri critici della traiettoria e, nell'eventualità di una interruzione, il tempo di azione dei retrorazzi necessario perché il veicolo potesse ricadere in uno dei punti prestabiliti come zone di recupero.

È noto quali siano le difficoltà di un simile calcolo, sopratutto tenendo conto, che in queste ipotesi, le equazioni del moto devono necessariamente tener conto della variazione della gravità con la quota, della azione di forze aereodinamiche che si sviluppano in condizioni supersoniche e ron densità dell'aria variabile, dell'azione del riscaldamento per attrito sui coefficienti di resistenza e di portanza del veicolo.

Si può dire che il calcolo dell'istante di azionamento dei retrorazzi e della relativa durata onde ottenere che il punto d'impatto cadesse in una delle zone prestabilite, fu il maggior lavoro che fu svolto dai calcolatori 7090 IBM del Goddard Center, tanto più che tutto il calcolo doveva essere eseguito nuovamente via via che veniva stabilita una nuova determinazione dei parametri orbitali.

In altro calcolo che, simultaneamente, doveva essere elfettuato era la determinazione sempre più approssimata delle variabili cinematiche, allo scopo di poter migliorare l'esattezza delle previsioni per le acquisizioni future. 
I calcolo svolti a questo scopo, erano basati sulla pisoluzione numerica di equazioni derivate dalle relazioni fondamentali di Newton. I metodi numerici impiegati consisterano nella integlazione numerica di Cowell per la estrapolazione e la correzione dei parametri orbitali e in correzioni differenziali per l'effetto delle perturbazioni minori.

Allo scopo di ridurre gli elfetti degli errori dovuti ai ladars e per tener conto delle muteroli caratteristiche atmosferiche, furono applicati pesi statistici ai dati provenienti dalle stazioni di rilevazione. In tal modo, la posizione del veicolo spaziale, relativamente a 18 settori terrestri, fu rontrollata costantemente e nel rodo più completo.

\section{I. CONCETTO DI PROGRAMMA MIONITOR.}

Ma, oltre alla diretta esecuzione dei calcoli un altro e forse più importante compito doveva essere svolto dai sistemi elettronici per l'elaborazione dei dati, ossia il controllo ed il coordinamento di tutti i calcoli the venivano via via eseguiti.

Infatti, in ogni istante, un dato calcolo doveva essere eseguito senza con ció, sacrificare qualche altro calcolo che arrebbe potuto essere di importanza determinante. Poteva, per esempio, accadere che un improvviso guasto di un'unita di uscita, compromettesse tutto il sistema di calcolo in tempo reale.

Per rendere interamente coordinato, in ogni istante ed in ogni eventualita, l'intero sistema di elabolazione venne studiato e messo a punto un apposito programma: il Monitor.

Il programma Monitor, caricato in memoria assieme ai programmi di calcolo, svolgeva una funzione di supervisione dello svolgimento dei singoli calcoli, coordinandone lo svolgimento secondo l'arrivo dei dati grezzi e la possibilità di emissione dei risultati, e stabilendo situazioni di priorità in base alle condizioni di entrata, di elaborazione e di uscita dei dati disponibili.

Come accade sovente, anche in questo caso la lisoluzione di un particolare problema tecnico, ha dato l'avvio ad una metodologia nuova la cui importanza trascende l'applicazione particolare che l'ha promossa.

it stata, in questa occasione, applieata per la prima volta una gerarchia concettuale di programmi di macchina: il programma Monitor che coordina il lavoro da eseguire, i programmi non-Monitor che eseguono il lavoro. 
En cosi diammico eoneetto di programmazione conduce a concepire un sistema per l'elaborazione dei dati come un'organizzazione in sé chiusa erl in grado di autodeteminarsi.

I mogrammi non-Monitor operano indipendentemente, ricevendo di rolta in rolta ordini dal Monitor in merito all'ar vio dei calcoli, all'indicazione di dove sono disponibili i dati da elaborate a di dove devono essere indirizzati i risultati oflenuti in modo tale che l'elaborazione, prò svolgersi in forma del tutto indipendente dalla configumaione del sistema claborante, e può continuare nonostante improrvise e casuali variazioni di questa.

\section{6. (ONSIDERMZIONI ('HENERATI.}

Jo'importanza ed i principali aspetti della elabolazione dettronica dei dati nei probleni del volo spaziale somo stati qui succintamente esposti nellal esemplificazione offerta dal progetto Mereury.

Ila è noto che non è questo l'mico esempio, né il più complesso. Ben altri problenni già presentano la necessità di una soluzione altrettanto efficiente nexgli studi e nei progetti, la cui meta è quella di passare dalla fase dei voli orbitali a quella, ben più ambiziosa, dei voli terra-luna.

Qui la necessita di calcolo in tempo reale, di raceolta e di smistamento veloce delle informazioni acquisite si propongono sotto una forma assai più ampla e complessa, si da richiedere teconiche a tecologie, ancolat più raffinate, ancol'a più avamzate.

In questi progetti è necessario inserire $u n$ opportuno centro di calcolo nel cuope del veicolo spaziale stesso, e far sì che anche questo sia in collegamento con ghli altri centri di calcolo del sistema, cosi che una ininterrotta rete di messaggi automatizzati comprenda in un unico campo la terra, la luma ed il veicolo spaziale.

Già sono stati realizzati modelli di clabomatori che usufruiscono delle tecniche di micro-miniaturizzazione.

Calcolatori elettronici, come il sistema IBAI tipo Thitan II, del peso (omplessivo di 90 libble a caparei di compiepe circa 150.000 operazioni al secondo, sperimentati sotto accelemazioni continue di 20 g. e urti di $100 \mathrm{~g}$.., in grado di svolgere il loro lavoro nelle condizioni più dispanate.

Su questi calcolatori, integrati da unità di telecommicazione ad alta volocità, i problemi del volo spaziale trovano la loro soluzione, cosi che gri astronauti di oggeri, ed ancor più quelli che seguiramo, possano disporre di quegli elementi che somo fondamentali per la sicurezza della propria vita e per il successo della propria missione. 